\title{
FLARES ON T TAURI STARS
}

\author{
G. F. GAHM \\ Stockholm Observatory \\ S-133 36 Saltsjöbaden \\ Sweden
}

\begin{abstract}
An overview of the characteristics of short-term light variability on $T$ Tauri stars is given. The evidence of the occurrence of flares comes from observations mainly at $X$ ray energies and from patrole observations in the ultraviolet spectral region. From such observations some limits on the peak fluxes and total energies of the largest flare-like events can be set. In addition, the frequency of such events can be deduced for a number of stars. It is demonstrated that there appears to be a qualitative difference between powerful flare-like events on the weak-line T Tauri stars (NTTs) and those on strong-line stars (CTTs). While it appears that the concept of surface flares occurring on NTTs may be correct there is the evidence that the disk-stars in addition produce flare-like events of a different nature. These events could be related to processes occurring not on the stars but in their circumstellar environment, for instance in a circumstellar disk. We also point at observations that could be of importance in clarifying the cause of flare-like activity on $\mathrm{T}$ Tauri stars and also comment on how this activity changes with stellar age.
\end{abstract}

\section{Introduction}

One characteristic property of $\mathrm{T}$ Tauri stars is that they vary in brightness with time. These variations occur on time-scales of minutes (or less) to years (or hundreds of years) and the pattern of variability may be drastically different from star to star, or even change with time for a given star. Regular as well as irregular fluctuations take place. Many of these properties were summerized by Herbig (1962) on the basis of existing observations in the optical spectral region. For reviews on more recent work concerning photometric variability of $T$ Tauri stars the reader is referred to Bouvier $(1986)$ and $\operatorname{Gahm}(1986,1988)$, for studies of UV-variability to Lago (1988), for studies of X-ray variability to Feigelson (1987) and Montmerle (1989) and for studies of radio-variability to André et al. (1988). T Tauri flare models have been reviewed by Giampapa (1986).

In the present review we will be concerned mainly with flare-like phenomena as observed mainly at optical wavelengths, phenomena that have been studied by several scientists, not least at the Burakan Observatory. The appearance of short-lived bursts of light on these stars and the possible relation to similar phenomena on flare-stars was a major topic already long ago (see e.g. Ambartzumian, 1957; Haro, 1976). Rather than to strive for a complete overview of the large number of papers in the field I have instead compiled some of the published and 
unpublished photometric data, sought to bring them together to see what we can learn about the propertics of flare-like phenomena on these stars.

\section{The Time-Scales of Flare-Like Events}

First we must agree on the definition of a flare-like event. In the present article I have taken the simple approach that a flare-like event occurs when the stellar light increases and decreases on time-scales similar to those of flares on flare stars and on the sun, that is over less than a few hours.The notion flare has often been attributed to light changes over timescales of days and months, but such variations are likely to be of a very different nature than for ordinary stellar flares. As will be evident below it is not even clear that all flare-like events, defined in the present way, are similar to flares seen on for instance flare stars.

Regular fluctuations on time-scales of days occur on several stars and it has been convincingly demonstrated that for many objects this pattern is related to the rotational modulation of dark and bright areas on the stellar surface (Bouvier et al., 1986; Hartmann et al., 1986). This behaviour is particularly striking for $\mathrm{T}$ Tauri stars with very weak optical emission-line spectra, sometimes referred to as naked T Tauri stars (NTTs). Also for a number of stars with stronger emission lines, classical T Tauri stars (CTTs), periods have been reported but here superimposed irregular variations make the identification of periods difficult. Repeated observations over long base-lines in time do not always confirm the periods found (Fischerström and Gahm, 1989) and a warning in accepting published periods for CTTs is in place.

\section{Observations of Flare-Like Events}

Most photometric work on $\mathrm{T}$ Tauri stars has been made for purposes other than to record flarelike events. Consequently, there is a huge reservoir of data collected with typically two or three observations per star and night. Obviously, if changes occur on a star during one night very little can be said about how these changes evolve. On the other hand, such data are most useful in setting limits to what the typical or what the most extreme changes are that occur on different stars, on different time-scales and in different wavelength bands. In fact, we will make use of observations containing only 3 observations or more for statistical purposes in Chapter 4.

Even when several observations are collected per night for a given star, the probability to catch both the rise and fall of a flare-like event lasting a few hours is small. It is in many cases difficult to judge what has actually passed. As an example we find in Fig. 1 (left) that the star The 12 during one night declined in 3 of the 4 filterbands of the Strömgren system (u centered at $3500 \AA, v$ at $4110 \AA, b$ at $4670 \AA$ and $y$ at $5470 \AA$ corresponding to the Johnson Vband). The slope resembles the declining part of a flare but whether there is a steep increase in flux just before the observations started or not can not be said. In Fig. 1 (right) is an example of an event taking place on the star RU Lup where possibly both the rise and the fall is observed. This is by our definition a flare-like event which is strong in all filterbands. It is, however, clear that the light curves have no resemblance to what is seen during flares on flare stars. While The 12 is an NTT, RU Lup is a CTT and it will be evident later that the probability to find an NTT in a phase of decline rather than rise, as in Fig. 1 a, is larger than for a CTT. 
mag.

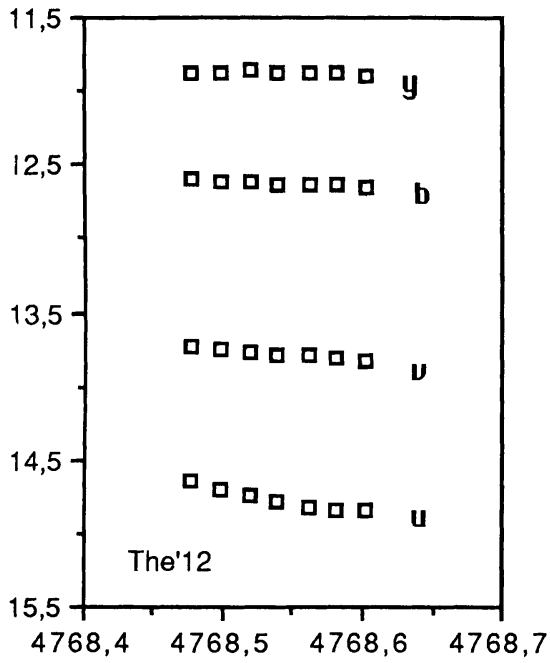

JD-2440000

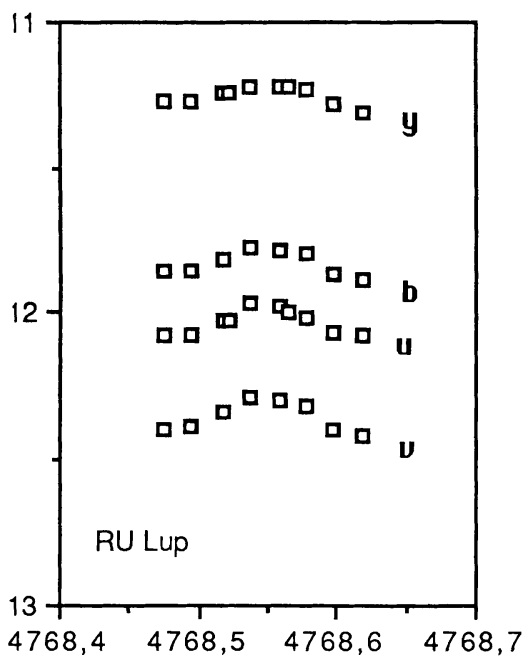

JD-2440000

Figure 1. left: Lightcurves of The 12 obtained over 3 hours of observations in the Strömgren $u$, $\mathrm{v}, \mathrm{b}$ and $\mathrm{y}$ filters when the star was decreasing in brightness in blue and violet light. right: A flare-like event observed on RU Lup.

\subsection{FREQUENCIES OF FLARE-LIKE EVENTS}

It has long been a rather wide-spread concept that $\mathrm{T}$ Tauri stars are very active and frequently undergo flare-like events. It is therefore rather disappointing to go through the literature and to find that major events, that is with rapid changes in the Johnson U-band of more than $20 \%$ in flux, are rather rare and that very few events for which both the rising and falling parts of the light-curve have been caught are at hand. A most striking example of such an event on T Tau itself has been found by Kilyachkov and Shevchenko (1976) but the authors state that during 4 sessions before and 15 sessions after the event no flares were found. On the basis of 750 hours of photographic patrole work in an area $5 \times 5$ degrees centered at R.A. $4^{\mathrm{h}}$ $30^{\mathrm{m}}$, Dec. $+24^{\circ}$ and covering numerous $\mathrm{T}$ Tauri stars 11 similar events, most of which reaching more than 1 magnitude above the quiescent level, were found by Hojaev (1987) on recognized T Tauri stars. Light-curves could be extracted for a few events but are not similar to those of stellar flares except for one event recorded on CI Tau (a CTT).

From the statistics made by Gahm (1986) on U-band variability on 22 stars over all together 886.5 hours of photometric patrole it was concluded that the fraction of time when the stars change in ultraviolet flux by more than $20 \%$ within 3 hours is 0.03 . This value is similar to the derived fraction of time with significant short-term $X$-ray variability $(0.05)$ but this does not mean that $X$-ray events and UV-events are related. Only very few events reach a total change in U-magnitude of $>1$ mag.

We can now compare these frequencies with what can be derived from the work by Hojaev (1987). About 50 T Tauri stars reside in the area according to the catalogue by Herbig and Bell 
TABLE 1. Frequencies of flare-like events.

Fraction of time when the stars

change in ultraviolet flux by

more than $20 \%$ within 3 hours

Fraction of time when major

outbursts occur $\left(\Delta \mathrm{U}>1.0, \Delta \mathrm{m}_{\mathrm{pg}}\right.$

$>0.7$ mag.)

Fraction of time with significant short-term X-ray variability

${ }^{*}$ Misprint in Gahm (1988)

(1988) and with 750 hours patrole time the total patrole time is 36750 hours. If the 11 events last for typically 3 hours then the flare frequency amounts to only 0.001 . Now, the events recorded with the photographic techniques are all very large $\left(\Delta \mathrm{m}_{\mathrm{pg}} \geq 0.7 \mathrm{mag}\right.$. and most events have $\Delta \mathrm{m}_{\mathrm{pg}}>1.0 \mathrm{mag}$.). The figure is consistent with the small number of major events in the compilation by Gahm (1986). Table 1 summerizes the findings where it should be noted that CTTs with strong emission lines are more frequently changing than those stars with weak emission lines.

Quite naturally one would expect much higher frequencies of events for which $\Delta U<20 \%$ and this is also evident in the works by Kuan (1976), Schneeberger et al. (1979 a and b) included in Worden et al. (1981), Shevschenko and Shutiomova (1981) and Zaitseva et al. (1985). More observations of "micro-flaring" is required to establish the properties of these small and sometimes rapid fluctuations.

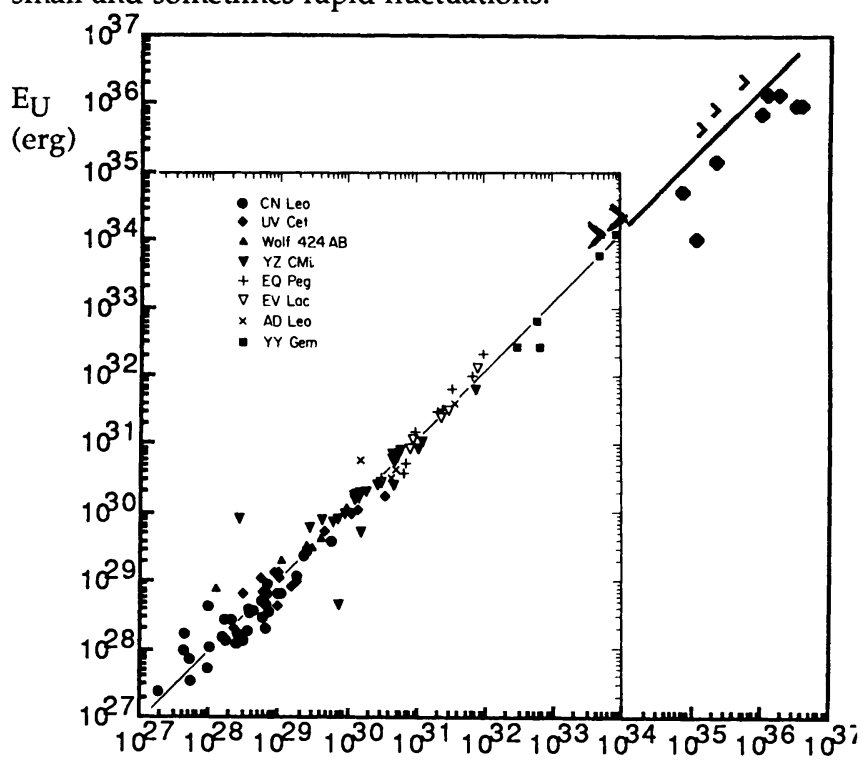

Figure 2. Total energies released in the Johnson $U$ - and $V$-band $\left(E_{U}\right.$ versus $E_{V}$ ) for flares on flare stars according to Lacy et al. (1976), inserted frame, and on T Tauri stars (upper right, where $>$ marks upper limits in $E_{V}$ ).

$$
E_{V}(e r g)
$$


$\mathrm{E}_{\mathrm{U}}$ (in $10^{35} \mathrm{erg}$ )

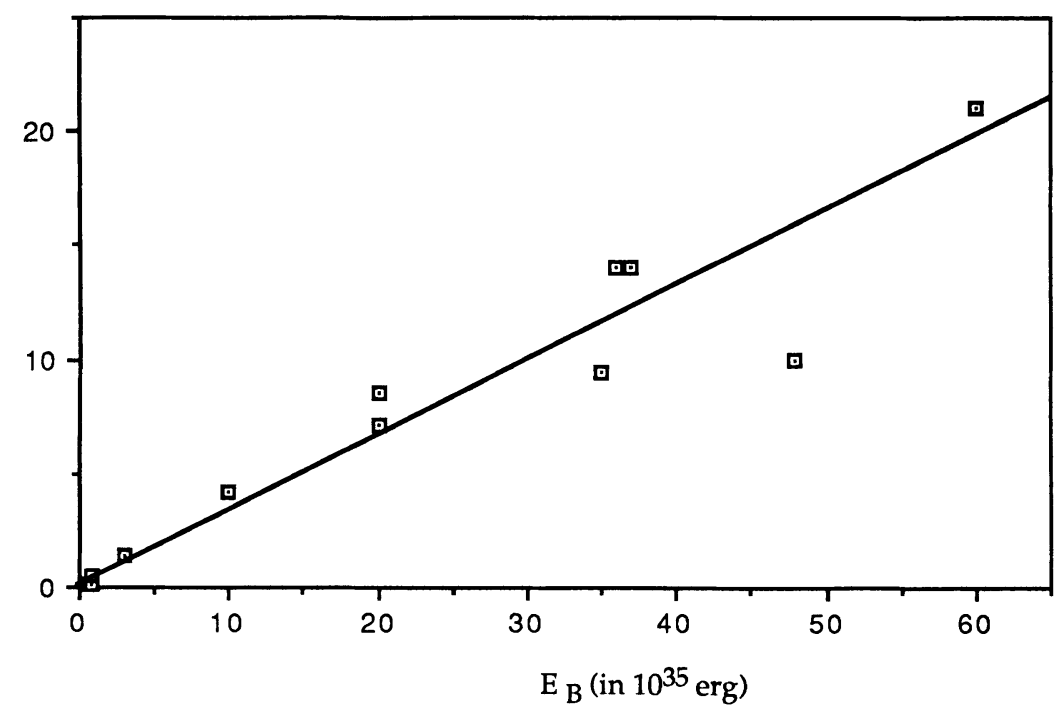

Figure 3. (above) Relation between energies $E_{U}$ and $E_{B}$ released during 13 flare-like events (4 events cluster at the lower left). The slope of the regression line is drastically different from that found for flare stars.

Figure 4. (right) Typical energy

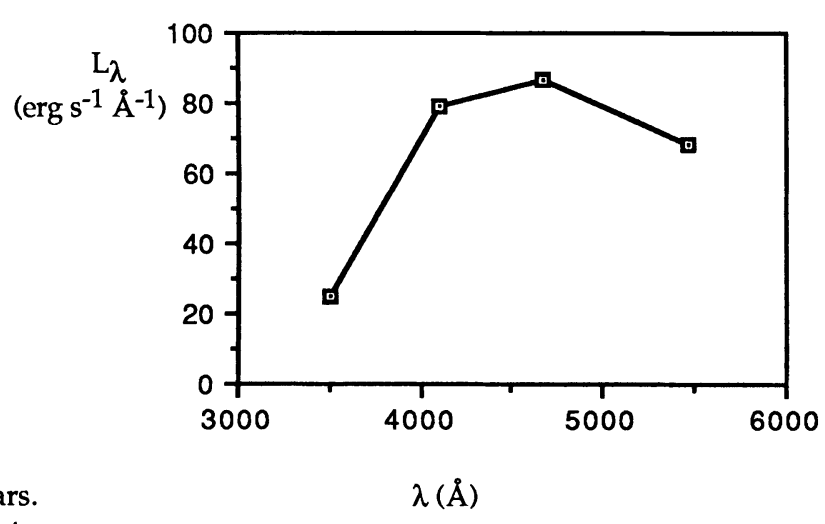
distribution at maximum phase of flare-like events on $\mathrm{T}$ Tauri stars. This particular case is for an event on RY Tau. Monochromatic luminosities (spherically symmetric case) are plotted versus wavelenghth after subtraction of background stellar flux.

\subsection{TOTAL ENERGIES RELEASED IN MAJOR EVENTS}

In the series of Strömgren photometric patrole made by C. Fischerström of the stars BP Tau, DI Tau, RY Tau, SU Aur, GW Ori, RU Lup and Thé 12 (= Sz 82) one can distinguish 13 flare-like events. It is possible to derive an estimate of the total energy released at the star in the 4 Strömgren filters (in several cases only as a upper limit). This analysis, which in part has been reported by Askebjer (1989) and in part is under preparation by Gahm (1989), leads to some interesting conclusions.

In Fig. 2 I have plotted the total energy released in the U-band (after proper correction for extinction and the difference in central wavelength and band-width relative to the $u$-band) against the total energy released in the V-band (with similar corrections) superimposed on the diagram showing the same quantities found for UV Ceti stars (Lacy et al., 1976). Not all 
flare-like events are detected in the V-band due to unfavourable contrast relative to the stellar background continuum. What is apparent in Fig. 2 is that the flare-like events registered are all more powerful than those of UV Ceti stars. In addition, even though the T Tauri events extend the relation found for flare stars, there are many events that fall below the straight line in Fig. 2. This effect is a significant one which will be apparent below.

All 13 events recorded in the $u$-band were also recorded in the b-band. Again, transformed into the Johnson U- and B-band a relatively close relation between the total energy released in $U$ and that released in $B$ is found in Fig. 3. The slope of the least-square line fit gives $E_{U}=$ $0.3 \mathrm{E}_{\mathrm{B}}$ to be compared to $\mathrm{E}_{\mathrm{U}}=1.20 \mathrm{E}_{\mathrm{B}}$ found for flare stars (Lacey et al., 1976). The flare-like events recorded on T Tauri stars are therefore different from flares on UV Ceti stars with regard to energy distribution and we will return to this issue in Section 3.3.

The total energies released in the $U$ band during these events range from $2 \cdot 10^{34}$ to $2 \cdot 10^{36} \mathrm{erg}$. Integrating over the optical spectral region (from Strömgren $u$ to $y$ ) the upper limit is $7 \cdot 10^{36}$ erg and possibly the very rare but extremely powerful events reported in Section 3.1 will reach $\sim 10^{38} \mathrm{erg}$, which provisionally can be taken as the upper limit of total energies released over the optical spectral region in flare-like events on $\mathrm{T}$ Tauri stars. This figure can be compared to the estimated lower limit of $8 \cdot 10^{35} \mathrm{erg}$ released during an extreme flare in X-rays on ROX 20 (Montmerle et al., 1983).

\subsection{THE ENERGY DISTRIBUTIONS}

Askebjer (1989) derived the energy distributions of the 13 events described above during maximum phase. One typical example is found in Fig. 4 . The energy distribution is drastically different from what is normally found for flares on flare stars in that the fluxes are much larger in blue than in ultraviolet light. The 13 events are very similar in this respect. The

TABLE 2. Characteristics of large flare-like events on T Tauri stars

$A$. Relations between the total energies released in $\bar{U}, \bar{B}$ and $V$

$\begin{array}{ll}\frac{\text { Flare stars }}{E_{\mathbf{u}}=1.20 \mathrm{E}_{\mathrm{B}}} & \frac{T \text { Tauri stars }}{\mathrm{E}_{\mathbf{u}}=0.3 \mathrm{E}_{\mathrm{B}}} \\ \mathrm{E}_{\mathbf{u}}=1.79 \mathrm{E}_{\mathbf{V}} & \mathrm{E}_{\mathbf{u}} \geq 0.6 \mathrm{E}_{\mathbf{V}}\end{array}$

B. Maximum energies released:

$E_{\mathbf{u}}=2 \cdot 10^{36} \mathrm{erg}$ (sample of 13 events)

$\left(\mathrm{E}_{\mathbf{u}}=2 \cdot 10^{34} \mathrm{erg}\right.$ for UV Ceti stars)

$\mathrm{E}_{\text {optical }}=7 \cdot 10^{36} \mathrm{erg}$ (possibly $\sim 10^{38} \mathrm{erg} \sim 0.1 \%$ of the time)

$\mathrm{E}_{\mathbf{X}} \sim 10^{36} \mathrm{erg}$

C. Maximum peak fluxes (sample of 13 events)

$\mathrm{F}_{\lambda}$ (Strömgren $\left.\mathrm{b}\right)=1.4 \cdot 10^{30} \mathrm{erg} \mathrm{s}^{-1} \AA^{-1}$

$\mathrm{F}_{\lambda}($ Strömgren $\mathrm{u})=2.3 \cdot 10^{29} \mathrm{erg} \mathrm{s}^{-1} \AA^{-1}$

D. Black-body temperatures derived at maximum light

$6200 \mathrm{~K}<\mathrm{T}<7300 \mathrm{~K}(13$ events $)$ 
distribution is not similar to emission from a hydrogen plasma. Rather, it fits in general quite well to a black-body law. When the corresponding black-body temperatures are derived they fall in a narrow range $7300 \mathrm{~K}>\mathrm{T}>6200 \mathrm{~K}$. The indication is that optical flare-like events on $\mathrm{T}$ Tauri stars are cool, much cooler than for those on flare stars. We will return to a discussion on these specific properties in Chapter 4 . The characteristics of large optical flare-like events on T Tauri stars, as derived in this chapter, are summerized in Table 2 where comparisons are made to flares on flare stars.

\section{Characteristics of the Brightness Changes}

We have seen in Chapters 2 and 3 that the light-curves of flare-like events on T Tauri stars are not always similar to what is found for flares on flare stars, that is with a rapid increase in flux followed by a slower decline. In order to further investigate this Askebjer (1989) and myself have made an inventory of published U-band measurements of T Tauri stars and included the unpublished Strömgren $\mathrm{u}$-band measurements by $\mathrm{C}$. Fischerström referred to above. A criterion has been that 3 or more observations were obtained per night and that an individual observer has observed the same star during several nights. Use is made also of corresponding measurements in $\mathrm{V}(=\mathrm{y})$. The data have been treated in the following way:

1. Both the $U$ (or $u$ ) and $V$ magnitudes are corrected for extinction, as a rule based on the values of $A_{V}$ given by Cohen and Kuhi (1979) or more recent determinations if avaible and on an average interstellar extinction curve; 2 . The relation between the so corrected $U$ and $V$ is plotted for each set of observations and as a rule the general slope can be described as linear with $\langle\mathrm{U}\rangle=\mathrm{C} \cdot\langle\mathrm{V}\rangle+$ const, where $\mathrm{C}$ is determined. These "gross" variations, in many cases related to periodic fluctuations, are then eliminated from the $U$ variations and only the residual changes in $U$ are extracted; 3 . For two consequtive observations during the same night the following quantities are determined: $\Delta U$ (or $\Delta u$ ) which is the change in magnitude after the above mentioned corrections and $\Delta t$ which is the time difference between the two measurements (see Fig. 5).

$\mathrm{u}$

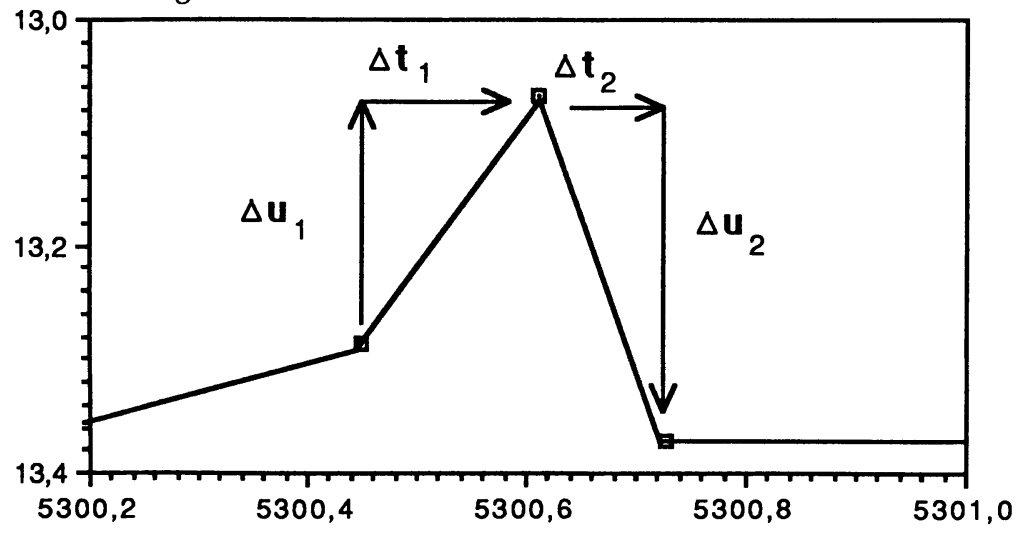

JD-2440000

Figure 5. The method for treating statistically how often and to what extent a star increases or decreases in brightness is based on measurements of magnitude difference $\Delta u$ and time difference $\Delta t$ between consecutive observations made during one night. The figure shows observations in u of RY Tau with only 3 observations this night. These provide 2 points in the $\Delta \mathrm{u}$ versus $\Delta t$ diagram (see Fig. 6). 
TABLE 3. References to photometric data for the stars selected

\begin{tabular}{llllll} 
Star & W(H $\alpha)$ & Ref. & Star & W(H $\alpha)$ & Ref. \\
& CTTs: & \multicolumn{3}{c}{ NTTs: } \\
RU Lup & 216 & $1,2,3$ & Thé 12 & 7 & 1 \\
DG Tau & 113 & 4,5 & SU Aur & 4 & $1,5,11,12,13$ \\
DI Cep & 95 & $1,6,7$ & WK 2 & 3 & 14,15 \\
TW Hya & 86 & 8,9 & V 410 Tau & 3 & $5,14,16,17$ \\
RW Aur & 84 & 1 & FK 2 & 2 & 5 \\
GW Ori & 46 & 1,5 & WK 1 & 2 & 14,15 \\
BP Tau & 40 & 5,10 & FK 1 & 2 & 5 \\
RY Tau & 20 & 1 & & &
\end{tabular}

References 1: Gahm (1989); 2: Bastian and Mundt (1979b); 3: Whittet et al. (1985); 4: Shaimieva and Shutiomova (1985); 5: Bouvier et al. (1989); 6: Grinin et al. (1980); 7: Kardopolov and Filip'ev (1985); 8: Rucinski and Krautter (1983); 9: Rucinski (1988); 10: Vrba et al. (1986); Pugach (1975); 12: Herbst et al. (1983); 13: Herbst et al. (1987); 14: Rydgren and Vrba (1983); 15: Rydgren et al. (1984); 16: Mozidse (1970); 17: Calvet et al. (1985).

The stars were selected as to make a comparison possible between CTTs and weak-line T Tauri stars (NTTs). In Table 3 the stars are listed according to stregth of H $\alpha$ emission with references to the works from which the photometric data were collected. A limit of $10 \AA$ of the equivalent width of $\mathrm{H} \alpha$ was set to separate strong-line and weak-line stars (so defining the two groups CTTs and NTTs).

Examples of diagrams showing $\Delta U(\Delta \mathrm{u})$ versus $\Delta t$ are given in Fig. 6. The orientation of $\Delta U$ is such that increases in fluxes are above the zero line. The probability that a given change is real and not due to noise increases in directions away from the zero line but is not always easy to estimate. We have, rather arbitrarily, introduced a level of noise at $\Delta u(\Delta U)= \pm 0.05$ mag. At any rate, points outside these limits have a large probability of representing real changes. What is striking is the general difference in pattern of rapid U-band variations on DI Cep and SU Aur in Fig. 6. The tendency that random observations of SU Aur preferentially selects the star in phases of decreasing brightness is present in all sets of observations, while DI Cep does not show any preference with regard to increasing or decreasing brightness. The former is a weak-line T Tauri star - the latter is a CTT.

We have gone through the whole material and simply computed the number of points below $\left(\mathrm{N}_{\mathrm{b}}\right)$ and number of points above $\left(\mathrm{N}_{\mathrm{a}}\right)$ the \pm 0.05 mag. levels and formed $\mathrm{N}_{\mathrm{b}} / \mathrm{N}_{\mathrm{a}}$ for different intervals in $\Delta t$. The results are summerized in Table 4 where the material is divided into CTTs and weak-line T Tauri stars (with the equivalent width of $H \alpha, W(H \alpha) \leq 10 \AA$ ) and for different intervals of $\Delta t$.

It appears to be a statistically significant difference between the two groups, especially when considering time-intervals in the range from $\Delta t=0.05$ to 0.1 Julian days ( 1.2 to 2.4 hours), which are rather typical time-scales of $\mathrm{X}$-ray flares on $\mathrm{T}$ Tauri stars and of strong flares on flare stars. In this interval there is a much larger probability to find a weak-line $\mathrm{T}$ Tauri star in a phase of declining than increasing brightness while the CTT has more or less equal probability to increase or decrease in brightness (as illustrated by the light-curves in Fig. 1). 
$\Delta \mathrm{u}$

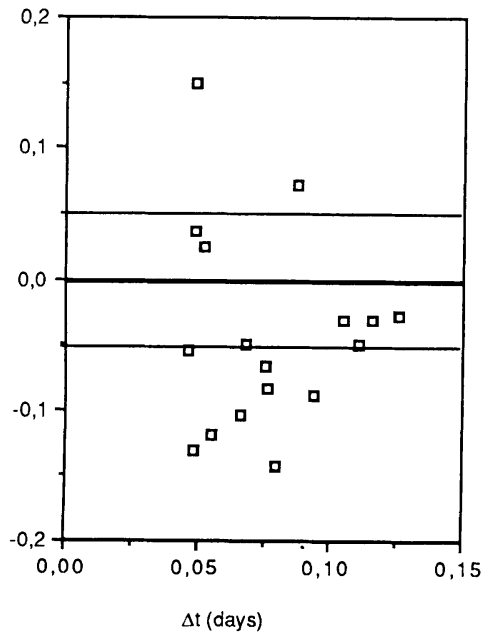

$\Delta \mathrm{U}$

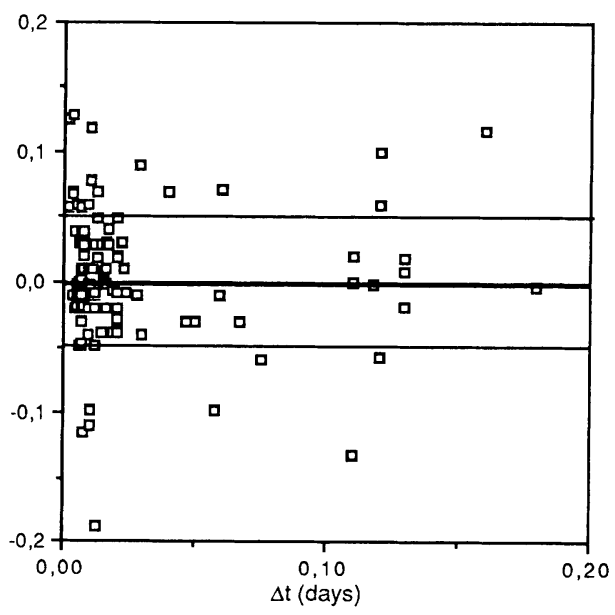

Figure 6. left: $\Delta \mathrm{u}$ versus $\Delta \mathrm{t}$ for a subset of Strömgren photometric data of SU Aur showing that statistically this star was observed more frequently in a phase of decreasing rather than increasing brightness. A rise in ultraviolet flux results in positive values of $\Delta \mathrm{u}$. right: $\Delta U$ versus $\Delta t$ for DI Cep showing no preference in the direction of the changes of brightness above the noise levels at \pm 0.05 mag.

TABLE 4. Statistics on direction of brightness changes on the stars selected

\begin{tabular}{lcc}
$\begin{array}{l}\text { Time interval } \\
\text { in JD }\end{array}$ & CTTs & $\mathrm{N}_{\mathrm{b}} / \mathrm{N}_{\mathrm{a}}$ \\
& & $\mathrm{NTTs}$ \\
$0<\Delta \mathrm{t}<0.05$ & 1.1 & 1.3 \\
$0.05<\Delta \mathrm{t}<0.1$ & 1.4 & 6.0 \\
$0.1<\Delta \mathrm{t}<0.2$ & 0.9 & 1.1 \\
\hline
\end{tabular}

The general pattern of rapid U-fluctuations on NTTs is therefore consistent with the presence of flares which occur relatively frequently. For the CTTs, on the other hand, the rapid Uband fluctuations are statistically of a totally different character. There is no reason to doubt that flares do occur on the CTTs as well, but for these stars it appears that changes of a different nature dominates the variations in the ultraviolet. We will return to a discussion on these findings in Chapter 5 . We close this chapter by noting that the difference found between the CTTs and NTTs is a statistical result including all stars. Some CTTs, notably RW Aur, have variability patterns more in line with the majority of the NTTs and some NTTs, notably V 410 Tau, behave similar to the CTTs. 


\section{Mechanisms}

Inspite of the large amount of photometric data available for $\mathrm{T}$ Tauri stars we still know rather little about the detailed properties of the flare-like events. In particular we lack simultaneous spectroscopic observations and good time-coverage at high photometric accuracy over the entire optical spectral region. From the results brought together in the preceding chapters one can put forward the following picture which hopefully can be tested by future observations:

1. T Tauri stars produce X-ray flares similar to those observed on flare stars but on occasion much more energetic.

2. Optical flares occur which are similar to flares seen on flare stars but sometimes much more energetic. It is unknown whether these flares are related to X-ray flares or not, although powerful U-band flares are as frequent as X-ray flares.

3. The strong optical flares have as a rule a different energy distribution than those of flare stars. For the former the indication is that the events are cool $\left(T_{\mathrm{BB}} \leq 8000 \mathrm{~K}\right)$. Less energetic events may have a different energy distribution, however.

4. The form of the light curves of strong optical flares on T Tauri stars is many times completely different than for typical flare star events, in particular for classical $\mathrm{T}$ Tauri stars where there is a slow rise followed by a slow decline in brightness. It is possible that on these CTTs, the strong events are of a completely different nature than stellar flares.

The present day data seem to indicate that ordinary flares over a large range of total energies occur frequently on $\mathrm{T}$ Tauri stars but that these events are masked by gross variations of a different kind on many CTTs (in particular). These stars are currently thought to be surrounded by circumstellar disks and it is natural to think that the flare-like events originate in the disk rather than on the stellar surface.

In the accretion model hypothesis developed by Shakura and Sunyaev (1973) and LyndenBell and Pringle (1974) and recently applied to T Tauri stars by e.g. Bertout et al. (1988) the rate of mass accretion, $\dot{\mathrm{M}}$, will lead to a luminosity of the boundary layer between the star and the disk of:

$$
\mathrm{L}=\mathrm{GM} * \dot{\mathrm{M}} /\left(2 \mathrm{R}_{*}\right)
$$

where $\mathrm{M} *$ and $\mathrm{R} *$ are the stellar mass and radius, respectively. It is then seen that a temporal increase in $\dot{M}$ over the boundary layer will produce an increase in the flux from the boundary layer, which could be one mechanism behind the peculiar flare-like events. However, the velocity of the flow of matter drifting inwards in the disk radially to the star is:

$$
\mathrm{v}_{\mathrm{r}}=-\dot{\mathrm{M}} /(2 \pi \mathrm{R} \Sigma)
$$

where $\mathrm{R}$ is the distance to an annulus of the disk and $\Sigma$ the surface density (see Pringle, 1981). In order to achieve time-scales of the order of a few hours a very thin boundary layer is required with current guesses on the surface density.

As is apparent in other articles in this volume, there is the possibility that the boundary layer of the disk is at a large distance from the stellar surface. Clearly, the peculiar flarelike events may be related to stellar surface flares behind large columns of gas in the line-ofsight. The events may simply be secondary phenomena in a circumstellar plasma that is heated by underlying flares and then left to cool. 
Figure 7. Rapid increase in $\mathrm{H} \alpha$ flux on DI Cep observed during one night by Bastian and Mundt (1979a). The equivalent width of $\mathrm{H} \alpha$ is plotted versus time.

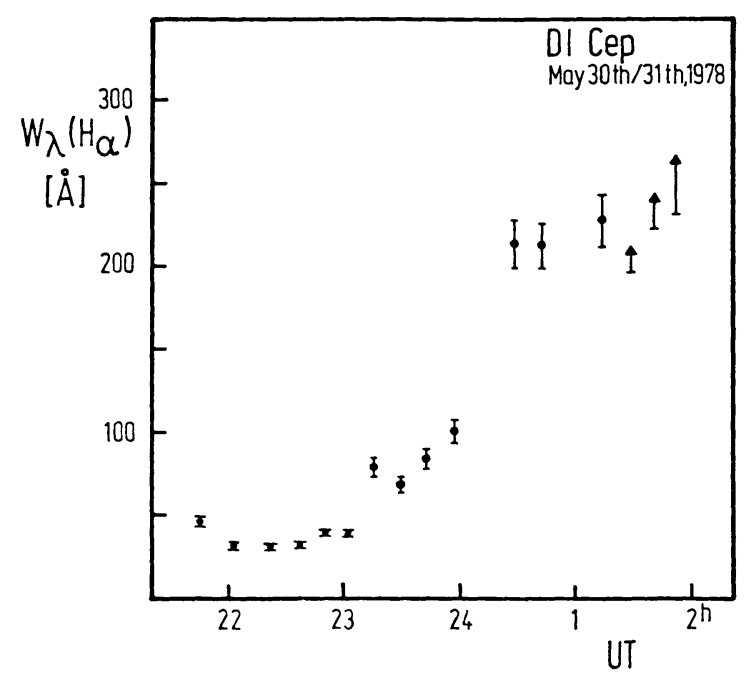

For the events that are similar to flares on flare stars one is directed to extrapolations of flare models, such as investigated by van den Oord (1987). Models of stellar flares are extrapolations of models of solar flares and these are still uncertain. An extrapolation of a solar flare model including an electric double layer (see Carlqvist, 1986) was made by Askebjer (1989) to account for the energetic flares on T Tauri stars.

\section{Further Aspects of Flares on Young Stars}

In this chapter we first want to draw the attention to observations of rapid variability of $T$ Tauri stars, observations that point at different processes than discussed so far. We will then end this overwiew by discussing how flare-like activity changes with age of the stars.

There are some reports on changes of profiles and/or intensities of emission lines on timescales of $<2$ hours. A dramatic example is shown in Fig. 7, where Bastian and Mundt (1979a) found the equivalent width of H $\alpha$ of DI Cep to increase by a factor of $\sim 4$ over 1 hour. They stated that significant variations of the strength of the emission lines occurred on this star on 2 of 6 observing nights on time-scales down to $\leq 15 \mathrm{~min}$.

Almost nothing is known about the relation between the broad-band events discussed above and the emission line changes. This is certainly an important area of future studies. For instance, if the major events are cool and not due to increased emission from a hydrogen plasma, will major changes nevertheless occur in the Balmer line fluxes? Can relations be found between U-band flux and Hy flux, such as has been found for flare stars (Butler et al., 1988)? Most certainly new insights to the physics and dynamics of the processes involved will be gained when line profiles are monitored simultaneously with the broad-band fluxes.

Another intriguing phenomenon is the rapid flips in colour observed by Gahm et al. (1989) on RY Lup when the star was faint. This star may be seen through the outer "fluffy" regions of a circumstellar disk and an interpretation is that when the star becomes occulted by foreground dust we see rapid phenomena in the disk or in a boundary layer. Do other stars show similar phenomena? 


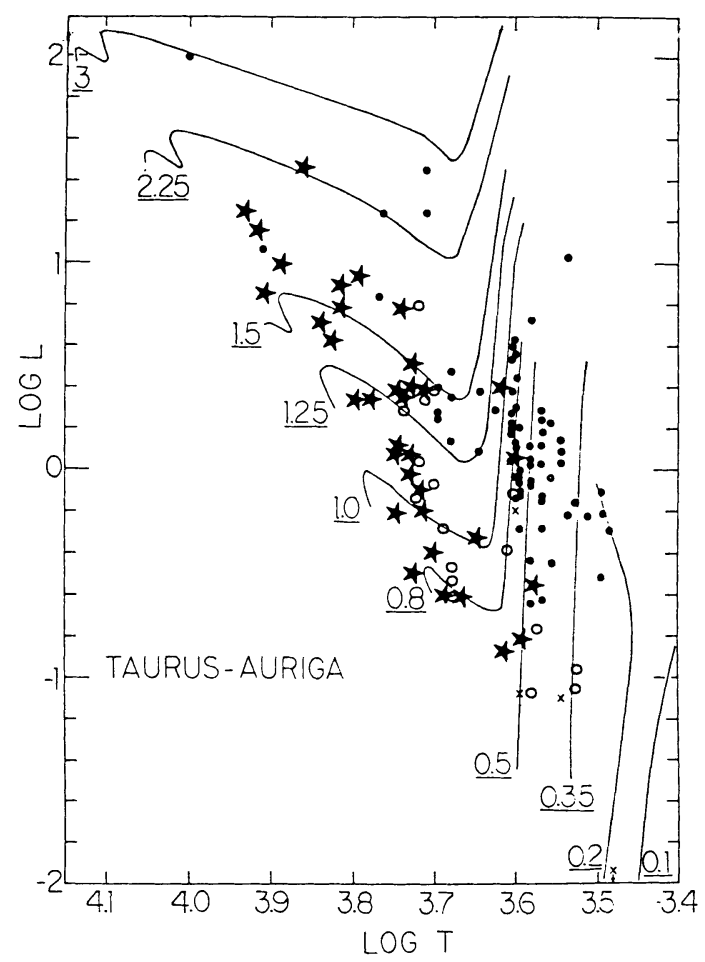

Figure 8. HR-diagram (luminosity versus effective temperature) where the dots represent the $T$ Tauri stars in the Taurus-Auriga region as plotted by Cohen and Kuhi (1979) with evolutionary model tracks for contracting stars of different mass. Superimposed are the post- $T$ Tauri candidates found by Lindroos (1986) - marked as stars - and by Walter et al. (1988) marked as open circles.

Turning now to the question of how flare activity changes with time as the stars evolve towards the main sequence we will focus at Fig. 8 as a starting point. In this HR-diagram we have plotted the post-T Tauri star candidates found by Lindroos (1986) from studies of young secondary components in double or multiple stars and those found by Walter et al. (1988) from $X$-ray surveys in the Taurus-Auriga region together with the $T$ Tauri stars in Taurus-Auriga according to Cohen and Kuhi (1979). The post-T Tauri candidates typically fall between the region with $\mathrm{T}$ Tauri stars and the main sequence. It is important to notice in relation to the discussion mentioned in Chapter 1 about the relation between $T$ Tauri stars and flare stars in young stellar aggregates, that the post- $T$ Tauri candidates represent a class of stars which is different from the ordinary flare stars. Most likely the late-type flare stars are derivatives of low-mass pre-main sequence objects most of which never had the high luminosities observed for the T Tauri stars in Fig. 8.

What can be said about the flare activity of the post-T Tauri stars, presumably representing ages between $5 \cdot 10^{6}$ years to zero-age main sequence age? In fact, very little is known about short-term variability on these stars. Tagliaferri et al. (1988) discovered a powerful X-ray flare, with a total energy of $10^{35} \mathrm{erg}$, on HD 560B - one of the stars in Lindroos' 
(1986) sample. This indicates that flares of $10^{4}$ times the energies liberated during prominent solar flares occur also after the $\mathrm{T}$ Tauri phase.

On the other hand, Feigelson (1989) reported that on the whole the X-ray post-T Tauri candidates seem to be more quiescent than the T Tauri stars. Furthermore, Hojaev (1989) has reported that during photographic patrole over a total of about 900 hours of observing time in the Taurus-Auriga field described in Chapter 3.1, no flares with $\Delta \mathrm{U}>0.2$ mag. were found on the (few) post-T Tauri candidates of Walter et al. (1988) in the field.

To summerize, we have a clear indication that the general degree of flare activity decays after $10^{7}$ years or so but also that very energetic flares may still occur. Certainly, this is an area to explore further by for instance monitoring post- $T$. Tauri stars photometrically over longer periods of time.

Finally, there is a cosmogonic connection to the question of flare activity evolution with age. If pre-main sequence solar mass stars are in a state of X-and/or U-flaring at levels of $10^{4}$ times the energy release of large solar flares during several percent of the time over $10^{8}$ years, it could mean that an early atmosphere of the planet Earth was exposed to very high doses of $X$-ray and ultraviolet radiation $\left(\sim 10^{9}\right.$ times that of the present quiescent sun) over a total period of several $10^{6}$ years. Although such fluxes may be of minor importance to the thermal history of our atmosphere it may be of great importance to the chemical evolution of the atmosphere.

\section{References}

Ambartsumian, V.A.: 1957, in "Non-stable stars", IAU symp. No 3 (ed. G.H. Herbig) p. 177

André, P., Montmerle, T., Feigelson, E.D., Stine, P.C., Klein, K.L.: 1988, In "Activity in cool star envelopes" (eds. O. Havnes, B.R. Pettersen, H.M.M. Scmitt, J.E. Solheim) Kluwer, p. 293

Askebjer, P.: 1989, Master Thesis (Stockholm Observatory, Sweden)

Bastian, U., Mundt, R.: 1979a, Astron. Astrophys. 78, 181

Bastian, U., Mundt, R.: 1979b, Astron. Astrophys. Suppl. 36, 57

Bertout, C., Basri, G., Bouvier, J.: 1988, Astrophys. J. 330, 350

Bouvier, J.: 1986, in "Protostars and molecular clouds" (eds. T. Montmerle, C. Bertout) Commissariat a l'Energie Atomique, France, p. 189

Bouvier, J., Bertout, C., Benz, W., M. Mayor, M.: 1986, Astron. Astrophys. 165, 110

Bouvier, J., Bertout, C., Boucher, P.: 1989, Astron. Astrophys. Suppl., in press

Butler, C.J., Rodono, M., Foing, B.H.: 1988, Astron. Astrophys. Lett. 206, L1

Calvet, N., Basri, G., Imhoff, C.L., Giampapa, M.S.: 1985, Astrophys. J. 293, 575

Carlqvist, P.: 1986, IEEE Transactions on plasma science, PS-14, p. 794

Cohen, M., Kuhi, L.V.: 1979, Astrophys.J. Suppl. 41, 743

Feigelson, E.D.: 1987, in "Protostars and molecular clouds" (eds. T. Montmerle, C. Bertout) Commissariat a l'Energie Atomique, France, p. 123

Feigelson, E.D.: 1989, private communication

Fischerström, C., Gahm, G.F.: 1989, preprint

Gahm, G.F.: 1986, In "Flares: Solar and Stellar", Rutherford Appleton Laboratory, RAL-86-085, p. 124

Gahm, G.F.: 1988, In "Formation and evolution of low mass stars" (Eds. A.K. Dupree, M.T.V.T. Lago) NATO ASI Series, p. 295

Gahm, G.F.: 1989, in preparation

Gahm, G.F., Fischerström, C., Liseau, R., Lindroos, K.P.: 1989, Astron. Astrophys. 211, 115

Giampapa, M.S.: 1986, In "Flares: Solar and Stellar", Rutherford Appleton Laboratory, RAL-86-085, p. 232 
Grinin, V.P., Efimov, Ju. S., Krasnobatsev, V.I., Shakhovskaya, N.I., Shcherbakov, A.G., Zaitseva, G.V.., Kolotilov, E.A., Shanin, G.I., Kiselev, N.N., Gjulaliev, Ch.G.,

Salmanov, I.R.: 1980, Peremennye Zvezdy (Variable Stars) 21, 247

Haro, G.: 1976, Bol. Inst. Tonantzintla 2, 3

Hartmann, L., Hewett, R., Stahler, S., Mathieau, R.D.: 1986 Astrophys. J. 309, 275

Herbig, G.H.: 1962, Advances Astron. Astrophys. 1, 47

Herbig, G.H., Bell, K.R.: 1988, Lick Obs. Bull., No 1111

Herbst, W., Holtzman, J.A., Klasky, R.S.: 1983, Astron. J. 88, 1648

Herbst, W., Booth, J.F., Koret, D.L., Zaitseva, G.V., Shakhovskaja, N.I., Vrba, F.J., Covino, E., Terranegra, L., Vittone, A., Hoff, D., Kelsey, L., Lines, R., Barksdale, W.: 1987, Astron. J. 94, 137

Hojaev, A.S.: 1987, Astrofizika (russ.) 27, 207

Hojaev, A.S.: 1989, private communication

Kardopolov, V.I., Filip'ev, G.K.: 1985, Peremennye Zvezdy (Variable Stars) 22, 103

Kilyachkov, N.N., Shevchenko, V.S.: 1976, Astron. J. USSR Lett. 2, 494

Kuan, P.: 1976, Astrophys. J. 210, 129

Lacy, C.H., Moffett, T.S., Evans, D.S.: 1976, Astrophys. J. Suppl. 30,85

Lago, M.T.V.T.: 1988, In "Formation and evolution of low mass stars" (Eds. A.K. Dupree, M.T.V.T. Lago) NATO ASI Series, p. 209

Lindroos, K.P.: 1986, Astron. Astrophys. 156, 223

Lynden-Bell, D., Pringle, J.E.: 1974, Mon. Not. Roy. Astron. Soc. 168, 603

Mosidze, L.N.: 1970, Bull. Obs. Abastumani 39, 21

Montmerle, T.: in "Low mass star formation and pre-main sequence objects" ESO-WS, in press Montmerle, T., Koch-Miramond, L., Falgarone, E., Grindlay, J.E.: 1983, Astrophys. J. 269,182 Pringle, J.E.: 1981, Ann. Rev. Astron. Astrophys. 19, 137

Pugach, A.F.: 1975, Peremmenye Zvezdy Prilozenie (Variable Stars Suppl.) 2, 195

Rucinsky, S.M.: 1988, Information Bull. Variable Stars (IAU), No. 3146

Rucinski, S.M., Krautter, J.: 1983, Astron. Astrophys. 121, 217

Rydgren, A.E., Vrba, F.J.: 1983, Astrophys. J. 267, 191

Rydgren, A.E., Zak, D.S., Vrba, F.J., Chugainov, P.F., Zaitseva, G.V.: 1984, Astron. J. 89, 1015

Schneeberger, T.J., Worden, S.P., Africano, J.L.: 1979a, Bull. American Astron. Soc. 11,439

Schneeberger, T.J., Worden, S.P., Africano, J.L.: 1979b, Information Bull. Variable Stars (IAU) No. 1582

Shaimieva, A.F., Shutiomova, N.: 1985, Peremennye Zvezdy (Variable Stars) 22, 176

Shakura, N.I., Sunyaev, R.A.: 1973, Astron. Astrophys. 24, 337

Shevchenko, V.S., Shutiomova, N.A.: 1981, Astrofizika (russ,) 17, 286

Tagliaferri, G., Giommi, P., Angelini, L., Osborne, J.P., Pallavicini, P.: 1988, Astrophys. J. Lett. 331, L 113

van den Oord, G.H.J.: 1987, "Stellar Flares", thesis (Utrecht, Holland)

Vrba, F.J., Rydgren, A.E., Chugainov, P.F., Shakovskaya, N.I., Zak, D.S.: 1986, Astrophys. J. 306, 199

Walter, F.M., Brown, A., Mathieau, R.D., Myers, P.C., Vrba, F.J.: 1988, Astron. J. 96, 297

Whittet, D.C.B., Davies, J.K., Evans, A., Bode, M.F., Robson, E.I., Banfield; R.M.: 1985, South African Obs. Circ. No 9, p. 55

Worden, S.P., Schneeberger, T.J., Kuhn, J.R., Africano, J.L.: 1981, Astrophys. J. 244, 520

Zaitseva, G.V., Kolotilov, E.A., Tarasov, A.E., Shenavrin, V.I., Shcherbakov, A.G.: 1985, Astron. J. USSR Lett. 11, 109 
MIRZOYAN: You have found that the ratios of energies in UBV regions of the spectrum are quite different. Is this because of the flares? If it is so then it can be assumed that it is a result of small number of flares observed in the case of $T$ Tauri stars. But if it is real difference, it could be explained by the observational fact that for flare stars we observe comparatively pure flare radiation while for $T$ Tauri stars it is a mixture of flare radiation and star radiation (flares on T Tauri stars do not usually begin from minimum level).

GAHM: My feeling is that the flare activity is high on both type of stars but that on the classical $T$ Tauri stars the rapid variations are dominated by this other process.

BENZ: It is interesting how low the temperature of $T$ Tauri flares is. Given the temperature and luminosity of a black body it is easy to calculate the size of the flare source. Can you tell me how big it is?

GAHM: I have not done this, but of course it is an easy thing to do and it should be made.

MONTMERLE: The distinction you see in $U$ band light curves for NTTS and CTTS is very interesting. Given that NTTS have no (hot) gas around them, and that CTTS on the contrary often have ionized winds with dM/dt up to about $10 * *(-8)$ solar masses per year, don't you think the differences might be due essentially to transfer effects of flare radiation through this circumstellar material?

GAHM: Yes definitely, this is certainly one possibility.

APPENZELLER: I would like to comment on your estimate of the minimum time scale of variations caused by changes of the accretion rate. These estimates obviously depend on the assumption about the size of the diskboundary zone (about one scale height?). As you know the physical nature and hence the extent of these layers are uncertain. In addition, the disks may not be stationary. If the boundary layer is very extended and if the matter is in free fall near the stellar surface, variations as short as a day or perhaps a few hours could be produced by variable accretion.

GAHM: That is interesting. I hope that observations of phenomena that may take place in the interior circumstellar regions of young stars can help to restrict the models. Of course we do not know the actual physical situation. But what is sure is that it is likely to be much more complicated than the simple models predict. 